\title{
Estimation of Treatment Benefit in Patients with Cerebral Arteriovenous Malformations
}

\author{
Peter Lindvall", Patric Blomstedt, A. Tommy Bergenheim \\ Department of Neurosurgery, Umeå University Hospital, Umeå, Sweden \\ Email: "peter_lindvall_nkk@hotmail.com
}

Received April 6, 2012; revised May 9, 2012; accepted June 3, 2012

\begin{abstract}
Background: Cerebral arteriovenous malformations (AVMs) are vascular lesions that may be associated with a significant morbidity and mortality. There is still today no consensus regarding treatment of unruptured AVMs. Using known data from the literature, and data from our own institution concerning stereotactic radiotherapy, our aim was to construct an equation that may be used to evaluate the benefit of intervention vs. conservative treatment in unruptured AVMs. Methods: Assuming an annual bleeding risk of $4 \%$ and previously reported mortality (29\%) and morbidity (16\%) rates after a bleeding from an AVM, an equation was constructed. This equation would estimate the time until the combined mortality and morbidity associated with conservative treatment would equal the mortality and morbidity of stereotactic radiotherapy. Results: Using stereotactic radiotherapy this treatment would benefit over conservative treatment in excess of 8.12 years. The risk of bleeding was the most important factor influencing the benefit of intervention vs. conservative treatment. Conclusions: We argue that that it may be possible to estimate the benefit of intervention vs. conservative treatment in an unruptured AVM. This may be achieved using our proposed equation and data specific to each center performing treatment for AVMs.
\end{abstract}

Keywords: Arteriovenous Malformation; Cerebrovascular Disease; Strokes; Treatment

\section{Introduction}

Cerebral arteriovenous malformations (AVMs) are thought to be congenital lesions consisting of a nidus with feeding arteries and draining veins [1]. Intracerebral haemorrhage (ICH) is the most common presenting symptom and occurs in the majority of patients before the age of 40. The mortality and morbidity rates following an ICH have previously been reported to be as high as $29 \%$ and $23 \%$, respectively [2]. Even if there are some inconsistent data concerning the risk of bleeding from an AVM, depending on factors such as angioarchitecture and previous haemorrhages, most authors have reported the annual risk to be $2 \%-4 \%$ [3-5]. While there is little controversy in treating AVMs that have bled there is no conclusive evidence concerning the benefit of treatment vs. conservative treatment in patients with non-ruptured AVMs. A Recent prospective study of patients with unruptured AVMs demonstrated a worse short-term outcome in treated vs. non-treated patients [6]. There are, however, difficulties in comparing two groups assigned for intervention or conservative treatment. Morbidity and mortality in the treated patients will depend on several factors such as treatment modality and the experience of

"Corresponding author. the respective center regarding the chosen treatment. The presented results concerning success rates, and morbidity/mortality related to the treatment have varied considerably in the literature, and the follow-up period has often been limited. Since complications of the treatment will most often occur in direct relation to the treatment, the follow-up time will be of outmost importance when comparing a treated and a non-treated group of patients.

Regarding these problems, we have developed mathematical equations which will allow the individual center to calculate the benefit of treating unruptured AVMs, with consideration to the temporal aspect, based on their own method of choice and results, provided that bleeding rates and morbidity/mortality for the non-treated group can be estimated. Our own material and data from the literature will be used as an example of how such calculations can be performed.

\section{Materials and Methods}

Equations were constructed for comparison of morbidity and mortality in treated and non-treated patients with cerebral AVMs (Table 1). The equations were then entered into the program MATLAB (MathWorks Inc.). The calculations will be presented below in a stepwise fashion, 
Table 1. Equations describing morbidity and mortality associated with conservative treatment vs treatment of AVMs.

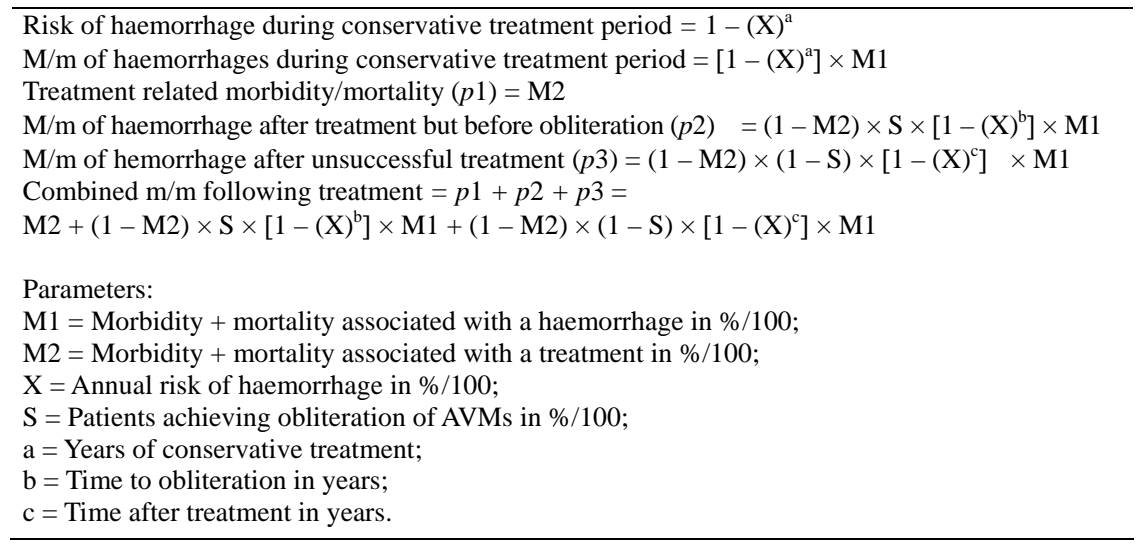

in an example based on our own material and data from the literature. The data concerning morbidity and mortality for treatment was collected from a recent study concerning hypofractionated stereotactic radiotherapy at Umeå University Hospital [7]. Figures concerning risk of bleeding and the mortality/morbidity associated with a haemorrhage were collected from the literature [2-4]. The risk of haemorrhage from an AVM was chosen to be $4 \%$ annually, mortality $29 \%$ and morbidity in surviving patients $23 \%$ for each bleeding, based on the study of Brown et al. [2]. A morbidity of $23 \%$ in surviving patients would correspond to $16 \%$ of the total population. Thus the combined morbidity and mortality for each haemorrhage would be $29 \%+16 \%=45 \%$.

The risk of bleeding over time (with an annual risk of $4 \%)$ was calculated using the equation proposed by Kondziolka et al. [8]: $1-(0.96)^{\text {years of remaining life }}$.

\section{Results}

The combined mortality and morbidity associated with a haemorrhage can be estimated using the following formula:

$$
\begin{aligned}
& \text { Life-time risk of haemorrhage } \\
& =1-(0.96)^{\text {years of remaining life }}
\end{aligned}
$$

Thus the risk of haemorrhage during the conservative treatment period is:

$$
\text { Risk of haemorrhage }=1-(0.96)^{\text {years of conservative treatment }}
$$

With a morbidity set at $16 \%$ and mortality at $29 \%$ the following formula describes haemorrhage associated morbidity and mortality during conservative treatment;

$$
\begin{gathered}
1-(0.96)^{a} \times(0.16+0.29) \\
(\text { Years of conservative treatment }=a)
\end{gathered}
$$

The combined morbidity/mortality associated with the natural course of an AVM will thus depend on the years of remaining life/number of years of conservative treatment.
In our experience of hypofractionated stereotactic radiotherapy of AVMs [7] the treatment related morbidity was $7.1 \%$, consisting of symptomatic radionecrosis. The bleeding risk for all treated patients before proven obliteration was $3.8 \%$ annually and two patients died of fatal haemorrhages before complete obliteration. The bleeding risk was close to the natural history of untreated AVMs and there was thus no excess treatment related mortality. The obliteration rate was $92.5 \%$ and the mean time until obliteration 3.2 years.

There are three possible scenarios that would lead to complications and unfavourable outcome following treatment; treatment related morbidity/mortality $(p 1)$, morbidity/mortality related to rupture of the AVM before proven obliteration ( $p 2)$, and morbidity/mortality related to treatment failure and the risk of haemorrhage associated with the natural history ( $p 3)$. This morbidity following treatment can be described as below:

Treatment related morbidity/mortality $(p 1)$ : The treatment related morbidity in our series was $7.1 \%$ whereas there was no treatment related mortality.

$$
p 1=0.071+0
$$

Morbidity/mortality related to a ruptured AVM before proven obliteration ( $p 2$ ): $92.5 \%$ of the patients reached obliteration, the risk of haemorrhage was set to $4 \%$, and the mean time to obliteration was 3.2 years.

$p 2=(1-0.071) \times(0.925) \times\left[1-(0.96)^{3.2}\right] \times(0.16+0.29)$

Morbidity/mortality related to a ruptured AVM upon unsuccessful treatment (p3): $7.5 \%$ of the patients did not reach obliteration.

$$
\begin{gathered}
p 3=(1-0.071) \times(0.075) \times\left[1-(0.96)^{c}\right] \times(0.16+0.29) \\
(c=\text { years after treatment })
\end{gathered}
$$

The combined morbidity and mortality following treatment would be the sum of $p 1+p 2+p 3$.

The following equation can then be constructed to describe how many years of conservative treatment needed to equal the morbidity of the treatment itself. The com- 
bined morbidity and mortality following a bleeding from an AVM is set at $45 \%$.

$$
\begin{aligned}
& {\left[1-(0.96)^{a}\right] \times(0.45) } \\
= & 0.071+(1-0.071) \times(0.925)\left[1-(0.96)^{3.2}\right] \times 0.45 \\
+ & (1-0.071) \times(0.075)\left[1-(0.96)^{c}\right] \times 0.45
\end{aligned}
$$

Calculations from MATLAB yields that $a=8.12$ (3)

Thus, in this example, using these assumptions and simple calculations it is obvious that slightly more than 8 years of conservative treatment will equal the morbidity of the chosen treatment. This equation has also taken in account the mortality and morbidity of haemorrhages during the latency period before obliteration, and in patients with treatment failure.

As seen above the time needed for treated related morbidity and mortality to equal the combined morbidity and mortality of conservative treatment will depend on several factors. Any change in bleeding rate, time to obliteration, obliteration rate and treatment related morbidity/mortality will affect the intersection between plotted morbidity/mortality for treatment vs. non-treatment. This is exemplified in Figures 1-4 where one parameter at the time was changed whereas all other parameters were the same as in the equation above. The mean time until obliteration was set at $3.2 \pm 0.5$ years (Figure 1), the obliteration rate was set in the interval $87.5 \%$ - 97.5\% (Figure 2), the bleeding was set at $4.0 \% \pm 2.0 \%$ (Figure 3) and the treatment related morbidity was set at $7.1 \% \pm$ $2.0 \%$ (Figure 4).

\section{Discussion}

Despite the fact that that AVMs were described by pathologists already in the middle of the nineteenth century [9] there still is some controversy regarding the benefit of intervention vs. conservative treatment, especially regarding nonruptured AVMs.

The aim of the current paper was to present simple mathematical equations that can be used by the individual department to calculate the benefits (or lack thereof) of treatment vs. non-treatment based on their own results. We have in this paper demonstrated the equations using estimations from the literature and our own results from stereotactic radiotherapy. Provided that our previous result have been correctly calculated, and that the estimations from the literature are reasonably correct, then at our department the treatment benefit seem to outweigh conservative treatment in excess of 8.12 years.

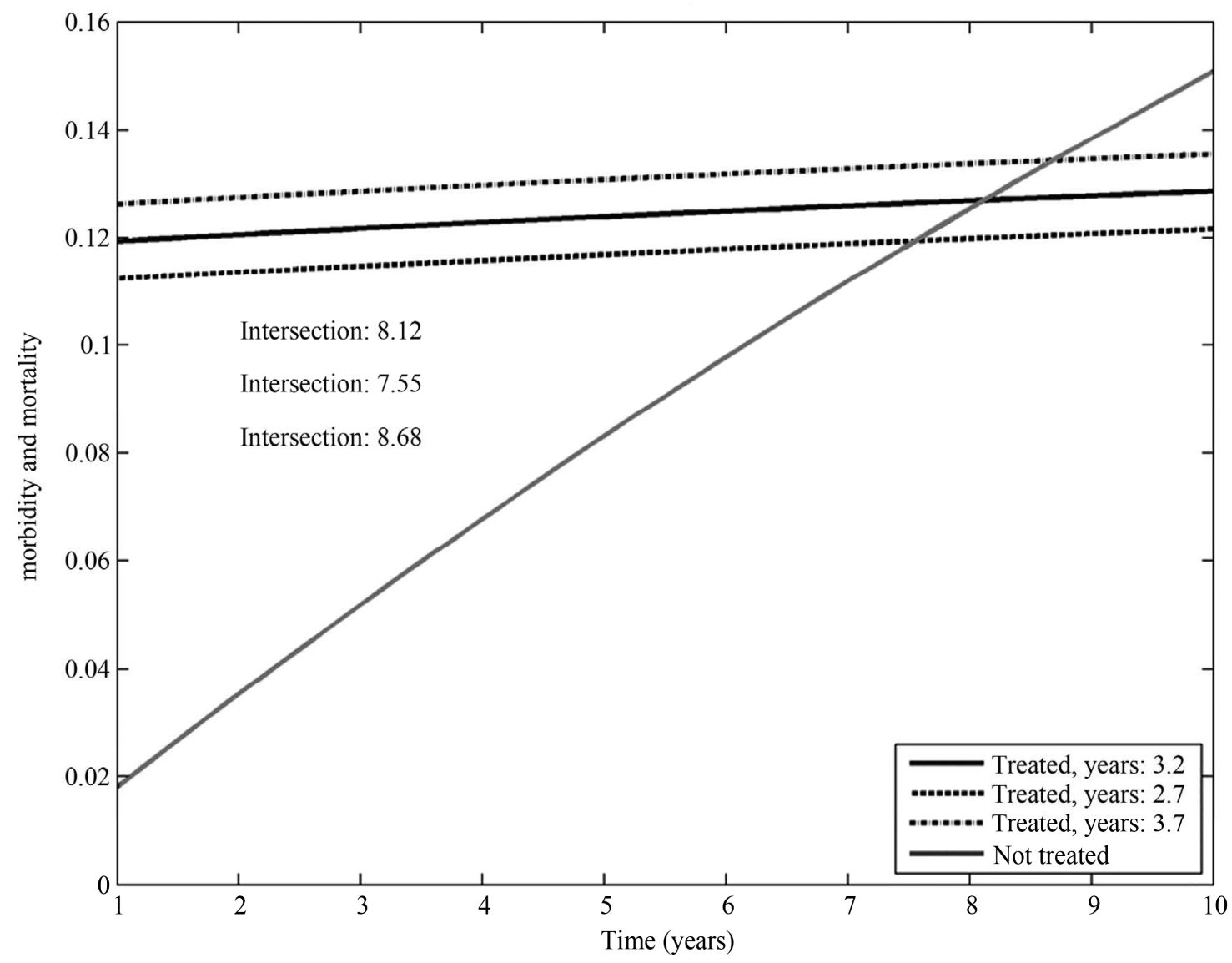

Figure 1. Combined morbidity and mortality in untreated and treated patients. The mean time until obliteration was set at $3.2 \pm 0.5$ years. 


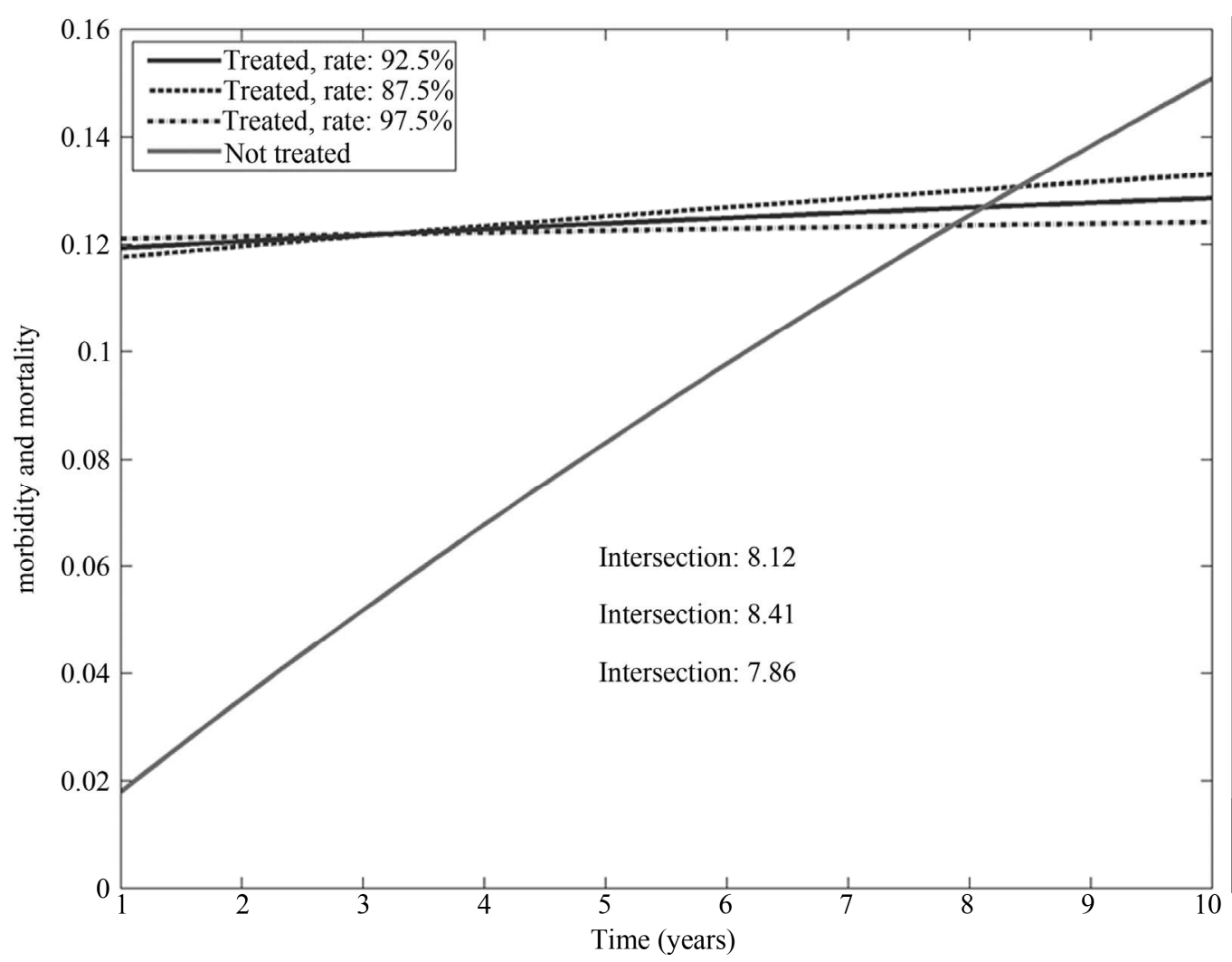

Figure 2. Combined morbidity and mortality in untreated and treated patients. The obliteration rate was set in the interval $92.5 \%-97.5 \%$.

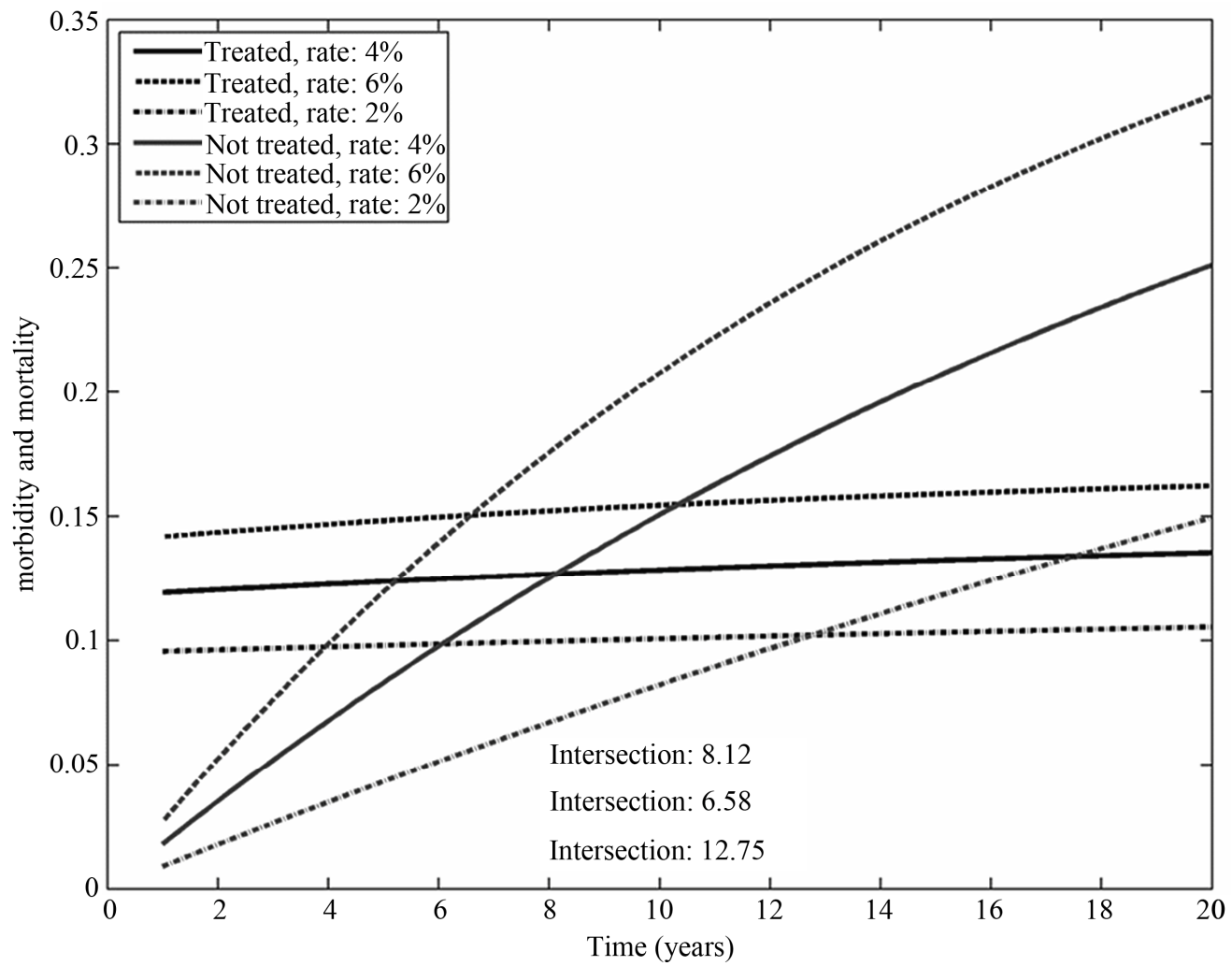

Figure 3. Combined morbidity and mortality in untreated and treated patients. The bleeding rate was set at $4.0 \% \pm 2.0 \%$. 


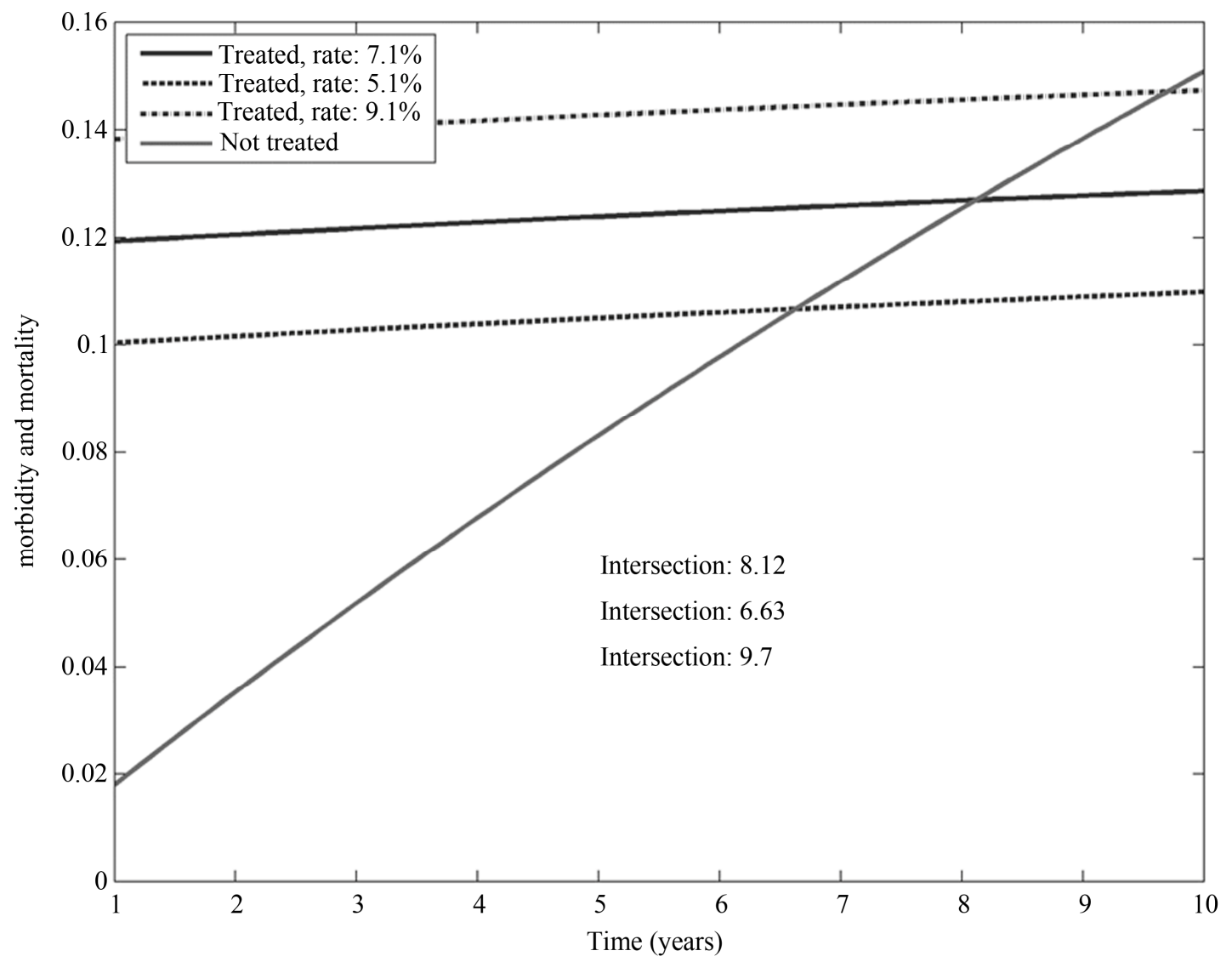

Figure 4. Combined morbidity and mortality in untreated and treated patients. The treatment related morbidity was set at $7.1 \% \pm 2.0 \%$.

Our material was however rather heterogeneous, consisting of various subgroups, the treatment underwent changes during the course of time, and some patients were lost to follow-up. For this reason the figure of 8.12 years should be regarded as a rough estimate, and is used only to demonstrate how to use the formula.

Unfortunately, the outcome from a mathematical equation will never be better than the quality of the data put into it. This does of course in this specific case apply not only to the effects of treatment, but also to the estimations from the literature regarding conservative treatment.

Various figures have been presented regarding bleeding rates and combined mortality and morbidity following an hemorrhage [10,11]. The $26 \%$ mortality reported by Brown et al. [2] and used in our example is for example considerably higher than the $6 \%$ reported in a recent paper [11]. The combined mortality/morbidity did however differ little between these studies ( $41 \%$ vs. $45 \%$ ), why the figure of $45 \%$ seems to be a reasonable estimation.

An annual bleeding rate of $2 \%$ - $4 \%$ would also seem as an adequate estimation. As seen from Figure 3 bleeding rate is the factor in our formula that would most strongly affect the intersection between intervention and conservative treatment. Assuming a bleeding rate of $2 \%$ annually would change the intersection to 12.75 years.

All these figures will depend on factors intrinsic to the treated AVMs as well as probably the experience and results of every center aiming to treat AVMs. The question is further complicated by the fact that we at present have access to three different forms of interventional therapies, which are likely to differ not only regarding efficacy and complications, but also regarding suitability in the individual patient. Partly for this reason we have hitherto not seen any truly randomized studies comparing the results of treatment vs. non treatment. The individual AVMs will not only differ regarding the inherent risk of rupturing and suitability for different treatments, but different centers are likely to exhibit various levels of success regarding the different treatments.

Microsurgical resection of AVMs with a Spetzler grade I-III has in experienced hands been reported to be associated with a low morbidity ranging from $0 \%-7 \%$ $[12,13]$. Surgical treatment will also have the benefit of instant cure, and not as in radiosurgical treatment, subject the patient to the risk of bleeding in the latency period until complete obliteration. For AVMs with a Spetzler 
grade of IV the morbidity has, however, been reported to substantially higher and was in one study reported to be 21.9\% [12]. The complication rate following embolisation has previously been reported to be as high as $40 \%$ [14] even if recent series have reported rates of mortality and permanent neurological morbidity ranging between $1.6 \%$ - 6.2\% [15,16]. Also concerning stereotactic radiosurgery different rates of success and complications have been reported. Obliteration rates are generally lower for large AVMs, and in one series the reported rate of obliteration was as low as $25 \%$ after 40 months in AVMs with a volume exceeding 15 cc [17]. Permanent treatment related morbidity is usually reported to be in the range of $3 \%-6 \%$ [18] even if this rate has been reported to be substantially higher in patients treated with a higher total dose of radiation [19].

The benefits of treatment vs. non-treatment can be easily calculated in theory with a reasonable degree of certainty. However, in reality the different parameters in the equation vary considerably between different groups of patients, treatments, centers and studies.

The figures presented above should therefore be regarded as a rough estimate as there are numerous factors that would affect this equation. We do however believe that such an evaluation of treatment related morbidity at each individual center, in relation to known data concerning bleeding rates and estimated mortality/morbidity may be helpful in determining treatment modality or if conservative treatment should be chosen for an individual patient.

We further believe that this estimation may provide some information regarding the timeframe needed to evaluate the benefit of treatment vs. conservative treatment.

A recent study comparing patients with treated AVMs and a group of patients conservatively treated showed that the treated patients had a worse functional outcome after 3 years [6]. Even if there are uncertain variables in the examples above, we can however with reasonable certainty conclude any study trying to compare conservative treatment vs. intervention should probably have a significantly longer time span than 3 years.

\section{Conclusion}

According to known data concerning the natural course of unruptured AVMs the combined morbidity and mortality over time may be significant. We argue that it may be possible to estimate treatment benefit in unruptured AVMs and suggest a mathematical equation for such a comparison. Using known data and data from our own institution treatment showed to be superior to conservative treatment in excess of 8.12 years. However, this figure must be seen as tentative, considering the uncertain- ties of the used parameters. When looking at the impact of different parameters on the benefit of treatment vs. non treatment, it does however seem evident that a considerable follow-up is essential for such estimations.

\section{Acknowledgements}

We are greatly indebted to Jon Bergenheim for valuable help concerning mathematics used to construct the equations and figures used in our paper.

\section{REFERENCES}

[1] H. J. Svien and J. A. McRae, “Arteriovenous Anomalies of the Brain. Fate of Patients not Having Definitive Surgery,” Journal of Neurosurgery, Vol. 23, No. 1, 1965, pp. 23-28. doi:10.3171/jns.1965.23.1.0023

[2] R. D. Brown Jr., D. O. Wiebers, G. Forbes, et al., "The natural History of Unruptured Intracranial Arteriovenous Malformations,” Journal of Neurosurgery, Vol. 68, No. 3, 1988, pp. 352-357. doi:10.3171/jns.1988.68.3.0352

[3] S. L. Ondra, H. Troupp, E. D. George, et al., "The Natural History of Symptomatic Arteriovenous Malformations of the Brain: A 24-Year Follow-Up Assessment,” Journal of Neurosurgery, Vol. 73, No. 3, 1990, pp. 387-391. doi:10.3171/jns.1990.73.3.0387

[4] B. E. Pollock, J. C. Flickinger, L. D. Lunsford, et al., "Factors that Predict the Bleeding Risk of Cerebral Arteriovenous Malformations,” Stroke, Vol. 27, No. 1, 1996, pp. 1-6. doi:10.1161/01.STR.27.1.1

[5] J. A. Hernesniemi, R. Dashti, S. Juvela, et al., "Natural History of Brain Arteriovenous Malformations: A LongTerm Follow-Up Study of Risk of Hemorrhage in 238 Patients,” Neurosurgery, Vol. 63, No. 5, 2008, pp. 823831. doi:10.1227/01.NEU.0000330401.82582.5E

[6] C. J. Wedderburn, J. van Beijnum, J. J. Bhattacharya, et al., "Outcome after Interventional or Conservative Management of Unruptured Brain Arteriovenous Malformations: A Prospective, Population-Based Cohort Study," Lancet Neurology, Vol. 7, No. 3, 2008, pp. 223-230. doi:10.1016/S1474-4422(08)70026-7

[7] P. Lindvall, P. Bergstrom, M. Blomquist, et al., "Radiation Schedules in Relation to Obliteration and Complications in Hypofractionated Conformal Stereotactic Radiotherapy of Arteriovenous Malformations," Stereotactic and Functional Neurosurgery, Vol. 88, No. 1, 2009, pp. 24-28. doi:10.1159/000260076

[8] D. Kondziolka, M. R. McLaughlin and J. R. Kestle, "Simple Risk Predictions for Arteriovenous Malformation Hemorrhage,” Neurosurgery, Vol. 37, No. 5, 1995, pp. 851-855. doi:10.1227/00006123-199511000-00001

[9] J. L. Pool, "Treatment of Arteriovenous Malformations of the Cerebral Hemispheres,” Journal of Neurosurgery, Vol. 19, No. 2, 1962, pp. 136-141.

[10] J. H. Choi, H. Mast, R. R. Sciacca, et al., "Clinical Outcome after First and Recurrent Hemorrhage in Patients with Untreated Brain Arteriovenous Malformation,” Stroke, Vol. 37, No. 5, 2006, pp. 1243-1247. 


\section{doi:10.1161/01.STR.0000217970.18319.7d}

[11] L. da Costa, M. C. Wallace, K. G. Ter Brugge, et al., "The Natural History and Predictive Features of Hemorrhage from Brain Arteriovenous Malformations,” Stroke, Vol. 40, No. 1, 2009, pp. 100-105. doi:10.1161/STROKEAHA.108.524678

[12] M. G. Hamilton and R. F. Spetzler, "The Prospective Application of a Grading System for Arteriovenous Malformations,” Neurosurgery, Vol. 34, No. 1, 1994, pp. 2-7. doi:10.1227/00006123-199401000-00002

[13] H. J. Pikus, M. L. Beach and R. E. Harbaugh, "Microsurgical Treatment of Arteriovenous Malformations: Analysis and Comparison with Stereotactic Radiosurgery," Journal of Neurosurgery, Vol. 88, No. 4, 1998, pp. 641-646. doi:10.3171/jns.1998.88.4.0641

[14] G. Wikholm, C. Lundqvist and P. Svendsen, "Embolization of Cerebral Arteriovenous Malformations: Part I, Technique, Morphology, and Complications,” Neurosurgery, Vol. 39, No. 3, 1996, pp. 448-459.

[15] V. Panagiotopoulos, E. Gizewski, S. Asgari, et al., "Embolization of Intracranial Arteriovenous Malformations with Ethylene-Vinyl Alcohol Copolymer (Onyx)," Ameri- can Journal of Neuroradiology, Vol. 30, No. 1, 2009, pp. 99-106. doi:10.3174/ajnr.A1314

[16] M. V. Jayaraman, M. L. Marcellus, S. Hamilton, et al., "Neurologic Complications of Arteriovenous Malformation Embolization Using Liquid Embolic Agents," American Journal of Neuroradiology, Vol. 29, No. 2, 2008, pp. 242-246. doi:10.3174/ajnr.A0793

[17] D. H. Pan, W. Y. Guo, W. Y. Chung, et al., “Gamma Knife Radiosurgery as a Single Treatment Modality for Large Cerebral Arteriovenous Malformations,” Journal of Neurosurgery, Vol. 93, No. 3, 2000, pp. 113-119.

[18] W. A. Friedman, F. J. Bova and W. M. Mendenhall, "Linear Accelerator Radiosurgery for Arteriovenous Malformations: The Relationship of Size to Outcome," Journal of Neurosurgery, Vol. 82, No. 2, 1995, pp. 180-189. doi:10.3171/jns.1995.82.2.0180

[19] E. Veznedaroglu, D. W. Andrews, R. P. Benitez, et al., "Fractionated Stereotactic Radiotherapy for the Treatment of Large Arteriovenous Malformations with or without Previous Partial Embolization,” Neurosurgery, Vol. 55, No. 3, 2004, pp. 519-531. doi:10.1227/01.NEU.0000134285.41701.83 